\title{
Monomeric CRP is Elevated in Patients with COPD Compared to Non-COPD Control Persons
}

\author{
Revathy Munuswamy',* \\ Jana De Brandt $\mathbb{D}^{2, *}$ \\ Chris Burtin ${ }^{2}$ \\ Wim Derave ${ }^{3}$ \\ Joseph Aumann ${ }^{4}$ \\ Martijn A Spruit $\mathbb{D}^{5,6, *}$ \\ Luc Michiels ${ }^{l, *}$
}

'Faculty of Medicine and Life Sciences, Biomedical Research Institute BIOMED, Hasselt University, Hasselt, Belgium; ${ }^{2}$ Faculty of Rehabilitation Sciences, Rehabilitation Research Center REVAL, Biomedical Research Institute BIOMED, Hasselt University, Hasselt, Belgium; ${ }^{3}$ Department of Movement and Sports Sciences, Ghent University, Ghent, Belgium; ${ }^{4}$ Department of Pneumology, Jessa Hospital, Hasselt, Belgium; ${ }^{5}$ Department of Research and Development, CIRO, Horn, the Netherlands; ' ${ }^{\circ}$ Department of Respiratory Medicine, Maastricht University Medical Centre, Faculty of Health, Medicine and Life Sciences, NUTRIM School of Nutrition and Translational Research in Metabolism, Maastricht, the Netherlands

*These authors contributed equally to this work

\begin{abstract}
Chronic low-grade systemic inflammation is frequently observed in patients with chronic obstructive pulmonary disease (COPD), e.g., elevated pentameric CRP (pCRP). However, pCRP can dissociate to form monomeric CRP (mCRP) which exhibits a clear proinflammatory behaviour in contrast to the more anti-inflammatory properties of pCRP. Therefore, mCRP may be an informative biomarker to demonstrate chronic low-grade systemic inflammation. This was confirmed by analysing serum samples from 38 patients with COPD and 18 non-COPD control persons (NCCP). mCRP was significantly elevated in patients with COPD vs. NCCP, indicating that $\mathrm{mCRP}$ might be considered as a new sensitive marker of chronic low-grade systemic inflammation.
\end{abstract}

Keywords: COPD, low-grade systemic inflammation, pCRP, mCRP

\section{Introduction}

Chronic low-grade systemic inflammation is frequently reported in patients with clinically stable chronic obstructive pulmonary disease (COPD). ${ }^{1}$ High-sensitive C-reactive protein, as measured in its native pentameric conformation (pCRP), is the most commonly measured inflammatory biomarker in research and clinical practice. However, it can irreversibly dissociate to form monomeric CRP (mCRP). ${ }^{2}$ These two CRP isoforms exhibit different biological functions. The complement pathway activation and low-density lipoprotein (LDL) metabolism are regulated more effective and versatile by mCRP than pCRP. ${ }^{2}$ Moreover, mCRP exhibits a strong pro-inflammatory behaviour, while pCRP has antiinflammatory properties. ${ }^{2}$ Therefore, mCRP may be a potential biomarker to demonstrate the presence of chronic low-grade inflammation. To our knowledge, mCRP has never been determined in patients with COPD. Hence, this study aimed to compare mCRP and pCRP between patients with clinically stable COPD and age/sex matched non-COPD control persons (NCCP). Additionally, clinical correlates of mCRP were studied in the patients. A priori, it was hypothesized that $\mathrm{mCRP}$ and $\mathrm{pCRP}$ are elevated in patients with COPD.

\section{Methods}

Parts of the baseline data of a prospective randomized controlled trial on the efficacy of a nutritional supplement in patients with COPD (NCT02770417) were analysed. Briefly, patients were recruited from June 2016 to November 2018 at the 
outpatient consultation of Jessa Hospital (Hasselt, Belgium) and were free of exacerbations $\geq 6$ weeks. Age/ sex matched NCCP (COPD:NCCP ratio 2:1) were recruited via advertisement. The study was approved by the Ethics Committees of Jessa Hospital (Hasselt, Belgium) and Hasselt University (Diepenbeek, Belgium) (Study registration number: B243201628086), and performed in accordance with the latest revision (2013) of the Declaration of Helsinki. All participants provided written informed consent prior to inclusion.

Age, sex, smoking status, number of respiratory hospitalizations 1 year before study enrolment, Charlson Comorbidity index, body mass index (BMI), fat mass index and lean mass index (fat mass/height ${ }^{2}$; lean mass/ height $^{2}$; DXA, lunar DPXL, General Electric Company, Boston, USA), spirometry data from NCCP (SpiroUSB, CareFusion, San Diego, USA; according to American Thoracic Society/European Respiratory Society (ATS/ ERS) guidelines), pulmonary function testing including post-bronchodilator spirometry, lung volumes and diffusion capacity for carbon monoxide (Master Screen Body and PFT, Jaeger, CareFusion, San Diego, USA; according to ATS/ERS guidelines) and Global Initiative for Chronic Obstructive Lung Disease (GOLD) stage from patients, impact of disease on daily life by COPD Assessment Test (CAT), grade of dyspnea by modified Medical Research Council (mMRC) scale, physical capacity (sixminute walk distance), and physical activity (PA) as steps per day, assessed via a tri-axial accelerometer (wGT3XBT, Actigraph, Pensacola, USA), were obtained.

Fasted venous blood samples were directly analysed in the Clinical Biology Laboratory of Jessa Hospital (Hasselt, Belgium) for pCRP with a Cobas 8000 modular analyzer (Roche, Basel, Suisse). Serum samples were stored at $-80^{\circ} \mathrm{C}$ (Biobank UBiLim) until analysis of mCRP, which was performed in a blind setup using the inhouse developed aptamer-based mCRP competition ELISA (patent pending: EP20212664.5, manuscript under preparation). Briefly, $\mathrm{COOH}$-coated plates (Biomat, TN, Italy) were activated by applying $0.4 \mathrm{M}$ of EDC and $0.1 \mathrm{M}$ of NHS in a 1:1 ratio. After immobilisation of pCRP $(2.5 \mu \mathrm{g} / \mathrm{mL})$ onto the activated plates, the unbound activated $\mathrm{COOH}$ surface groups were blocked using 5\% PBS-marvel and $1 \mathrm{M}$ ethanolamine, $\mathrm{pH} 8$ overnight and 1 hour respectively. Next, in situ pCRP monomerization was achieved by applying $10 \mathrm{mM} \mathrm{NaOH}$ at room temperature (RT) for $30 \mathrm{~min}$. Twenty-five $\mu \mathrm{L}$ of serum sample was incubated with $150 \mathrm{nM}$ biotin labelled aptamer on a rotating mixer for $30 \mathrm{~min}$ at RT and then transferred to a mCRP coated plate for $30 \mathrm{~min}$ at $37^{\circ} \mathrm{C}$ on shaker at $400 \mathrm{rpm}$. After incubation, the wells were washed three times and incubated on a shaker at $400 \mathrm{rpm}$ with SA-HRP (Life Technologies, Belgium). The enzyme-substrate colour reaction was then stopped by adding a stop solution $\left(0.18 \mathrm{M} \mathrm{H}_{2} \mathrm{SO}_{4}\right)$ to the wells and the standards and samples were measured at $450 \mathrm{~nm}$ wavelength using a MultiskanTM FC Microplate Absorbance Reader (Thermo Scientific, Belgium).

For statistical analysis SPSS version 24.0 (SPSS Inc., Chicago, USA) was used. Results are described as mean \pm standard deviation or median (quartile 1-quartile 3), as appropriate. Proportions are expressed in percentages and comparison between groups was performed via Chi Square test for homogeneity or Fisher's test, as appropriate. All characteristics, pCRP and mCRP were compared between groups by using Independent $T$-test or Mann-Whitney $U$-test, as appropriate, and a $P$-value $\leq 0.05$ was used for significance. Two sub-analyses were performed: i) comparison of mCRP between COPD and NCCP after excluding persons with a $\mathrm{pCRP}>3 \mathrm{mg} / \mathrm{L}$; ii) comparison of mCRP and pCRP between COPD ex-smokers and COPD smokers, and between NCCP non-smokers and NCCP exsmokers. Spearman's rho correlation coefficients were determined (rho $<0.3=$ weak correlation; $0.3-0.7=\bmod -$ erate correlation; $>0.7=$ strong correlation). A $P$-value of $\leq 0.01$ was used for significance to correct for multiple correlation testing.

\section{Results}

Serum samples were available from 38 patients $(65 \pm 6$ years, $74 \%$ male, BMI: $25.8 \pm 4.7 \mathrm{~kg} / \mathrm{m}^{2}$, forced expired volume in $1 \mathrm{sec}\left(\mathrm{FEV}_{1}\right): 55.6 \pm 14.0 \%$ predicted), who typically had moderate-to-severe airflow obstruction, static hyperinflation and decreased diffusion capacity, moderate symptom burden, and a median of two comorbidities. Most patients (97\%) were (ex)-smoker. Physical capacity was quite well preserved, while patients were generally physically inactive (Table 1). Moreover, 18 NCCP (65 \pm 6 years, $78 \%$ male, BMI: $26.4 \pm 3.0 \mathrm{~kg} / \mathrm{m}^{2}, \mathrm{FEV}_{1}: 104.8 \pm 10.2$ $\%$ predicted) were analysed. Patients with COPD had a similar body composition compared to NCCP (Table 1).

Median pCRP was higher in patients with COPD (1.85 (1.05-4.20) $\mathrm{mg} / \mathrm{L}$ versus 0.75 (0.30-2.18) $\mathrm{mg} / \mathrm{L}$ in NCCP; $P=0.013$; Figure 1A). Median mCRP levels were higher in patients with COPD (0.66 (0.38-1.03) $\mathrm{mg} / \mathrm{L})$ versus NCCP (0.00 (0.00-0.29) mg/L; $P<0.001 ;$ Figure 1A). 
Table I Baseline Characteristics of Patients with COPD and Correlation Coefficients with mCRP and PCRP

\begin{tabular}{|c|c|c|c|c|}
\hline Characteristics & Patients with COPD $(\mathbf{N}=38)$ & $\begin{array}{c}\text { Non-COPD } \\
\text { Control Persons } \\
(\mathbf{N}=18)\end{array}$ & $\begin{array}{l}\text { Correlation with } \\
\text { mCRP (rho) }\end{array}$ & $\begin{array}{l}\text { Correlation with } \\
\text { pCRP (rho) }\end{array}$ \\
\hline Age (y) & $65 \pm 6$ & $65 \pm 6$ & 0.162 & 0.122 \\
\hline $\operatorname{Sex}(\mathrm{N}[\% \mathrm{male}])$ & $28[74]$ & $14[78]$ & - & - \\
\hline BMI $\left(\mathrm{kg} / \mathrm{m}^{2}\right)$ & $25.8 \pm 4.7$ & $26.4 \pm 3.0$ & -0.200 & 0.040 \\
\hline $\mathrm{BMI} \geq 30 \mathrm{~kg} / \mathrm{m}^{2}$ (N [\%]) & $7[18]$ & $2[11]$ & - & - \\
\hline Fat mass index $\left(\mathrm{kg} / \mathrm{m}^{2}\right)^{\dagger}$ & $7.2(5.4-9.1)$ & $6.6(6.1-8.5)$ & -0.281 & -0.010 \\
\hline Lean mass index $\left(\mathrm{kg} / \mathrm{m}^{2}\right)^{\dagger}$ & $18.6(15.7-20.1)$ & $19.4(17.7-20.0)$ & -0.115 & 0.000 \\
\hline FEV (\%predicted) & $55.6 \pm 14.0^{*}$ & $104.8 \pm 10.2$ & 0.213 & -0.017 \\
\hline $\mathrm{FEV}_{\mathrm{l}} / \mathrm{FVC}(\%)$ & $50.5 \pm 11.9 *$ & $73.2 \pm 6.1$ & 0.189 & -0.259 \\
\hline TLC (\%predicted) & $117.3 \pm 16.2$ & - & -0.134 & -0.078 \\
\hline RV (\%predicted) & $177.7 \pm 40.6$ & - & -0.244 & -0.293 \\
\hline DLCO SB (\%predicted) & $52.3(44.6-63.3)$ & - & 0.085 & -0.121 \\
\hline GOLD Stage: I, II, III, IV (N [\%]) & 3 [8], 21 [55], 13 [34], I [3] & - & - & - \\
\hline $\begin{array}{l}\text { Hospitalization in last year: } 0, \mathrm{I},>\mathrm{I} \\
(\mathrm{N}[\%])\end{array}$ & 32 [84], 5 [13], I [3] & 18 [100], $0[0], 0[0]$ & - & - \\
\hline Smoking: NS, EX, S (N [\%]) & $\mathrm{I}[3], 2 \mid[55], \mathrm{I} 6[42]^{*}$ & 7 [39], II [6I], 0 [0] & - & - \\
\hline mMRC (points) & $\mathrm{I}(0-1)^{*}$ & $0(0-0)$ & -0.322 & -0.054 \\
\hline CAT (points) & $14 \pm 6^{*}$ & $5 \pm 4$ & -0.271 & -0.152 \\
\hline $\mathrm{CCl}$ (points) & $2(I-3)^{*}$ & $0(0-1)$ & -0.274 & 0.086 \\
\hline 6MWD (m) & $505 \pm 76^{*}$ & $664 \pm 64$ & 0.193 & -0.012 \\
\hline $6 \mathrm{MWD}<357 \mathrm{~m}(\mathrm{~N}[\%])$ & $0[0]$ & $0[0]$ & - & - \\
\hline PA (steps/day) $)^{\ddagger}$ & 4499 (3402-8198)* & 7024 (6505-9198) & 0.004 & -0.128 \\
\hline $\mathrm{PA}<5000$ steps $/$ day $(\mathrm{N}[\%])^{\ddagger}$ & $19[56]^{*}$ & I [6] & - & - \\
\hline mCRP (mg/L) & $0.66(0.38-1.03)^{*}$ & $0(0-0.29)$ & - & 0.076 \\
\hline PCRP (mg/L) & $1.85(1.05-4.20)^{*}$ & $0.75(0.30-2.18)$ & 0.076 & - \\
\hline
\end{tabular}

Notes: rho $=$ Spearman correlation coefficient, rho $<0.3=$ weak correlation; rho $=0.3-0.7=$ moderate correlation; rho $>0.7=$ strong correlation. $*$ Significant $P<0.05$; ${ }^{\dagger}$ Sample size is $n=37$ patients with COPD for this outcome; ${ }^{\ddagger}$ Sample size is $n=34$ patients with COPD for this outcome.

Abbreviations: COPD, Chronic Obstructive Pulmonary Disease; mCRP, monomeric C-reactive Protein; PCRP, native pentameric C-reactive Protein; BMI, Body Mass Index; FEV , Forced Expired Volume in one second; FVC, Forced Vital Capacity; TLC, Total Lung Capacity; RV, Residual Volume; DLCO SB, Diffusion capacity of the Lung for Carbon Monoxide Single Breath; GOLD, Global initiative for Chronic Obstructive Lung Disease; NS, Non-Smoker; EX, EX-smoker; S, Smoker; mMRC, modified Medical Research Council scale for dyspnea; CAT, COPD Assessment Test; CCI, Charlson Comorbidity Index; 6MWD, Six-Minute Walking Distance; PA, Physical Activity. y, years; $\mathrm{N}$, number; kg, kilogram; $\mathrm{m}$, meter; \%, percentage; $\mathrm{mg} / \mathrm{L}$, milligram per litre.

After excluding all participants with a pCRP $>3 \mathrm{mg} / \mathrm{L}$ (COPD, $n=12$ and NCCP, $n=3$ ), median mCRP was still higher in patients: $0.61(0.38-0.90) \mathrm{mg} / \mathrm{L}$ versus 0.00 $(0.00-0.28) \mathrm{mg} / \mathrm{L}$ in NCCP $(P<0.001)$. pCRP and mCRP were not different between COPD smokers and COPD exsmokers, and between NCCP non-smokers and exsmokers (Figure 1B). pCRP and mCRP did not correlate with each other nor with any of the other patients' clinical characteristics (Table 1).

\section{Discussion and Conclusion}

Systemic mCRP levels were increased in patients with clinically stable COPD and did not correlate with pCRP or clinical characteristics. pCRP is a diagnostic biomarker for COPD exacerbation ${ }^{3}$ and a prognostic biomarker for early mortality. ${ }^{4}$ However, pCRP may not reveal the complete role of CRP in chronic low-grade systemic inflammation. On binding with liposomes, cell membranes and activated platelets through lysophosphatidylcholine, pCRP dissociates into mCRP subunits revealing new epitopes, which is known to have a more potent proinflammatory effect in contrast to pCRP. This mCRP isoform is typically elevated during early low-grade chronic inflammation. ${ }^{2}$ This explains the current results, as the patients with COPD had a substantial increase in mCRP levels, while mCRP was not detectable in $70 \%$ of the NCCP. The difference in mCRP between COPD and NCCP remained after excluding all participants with an increased pCRP level $(>3 \mathrm{mg} / \mathrm{L})$. Also, smoking was found to be non-discriminating within patients with COPD or within NCCPs for pCRP and mCRP. Therefore, our results provide a clear indication that 

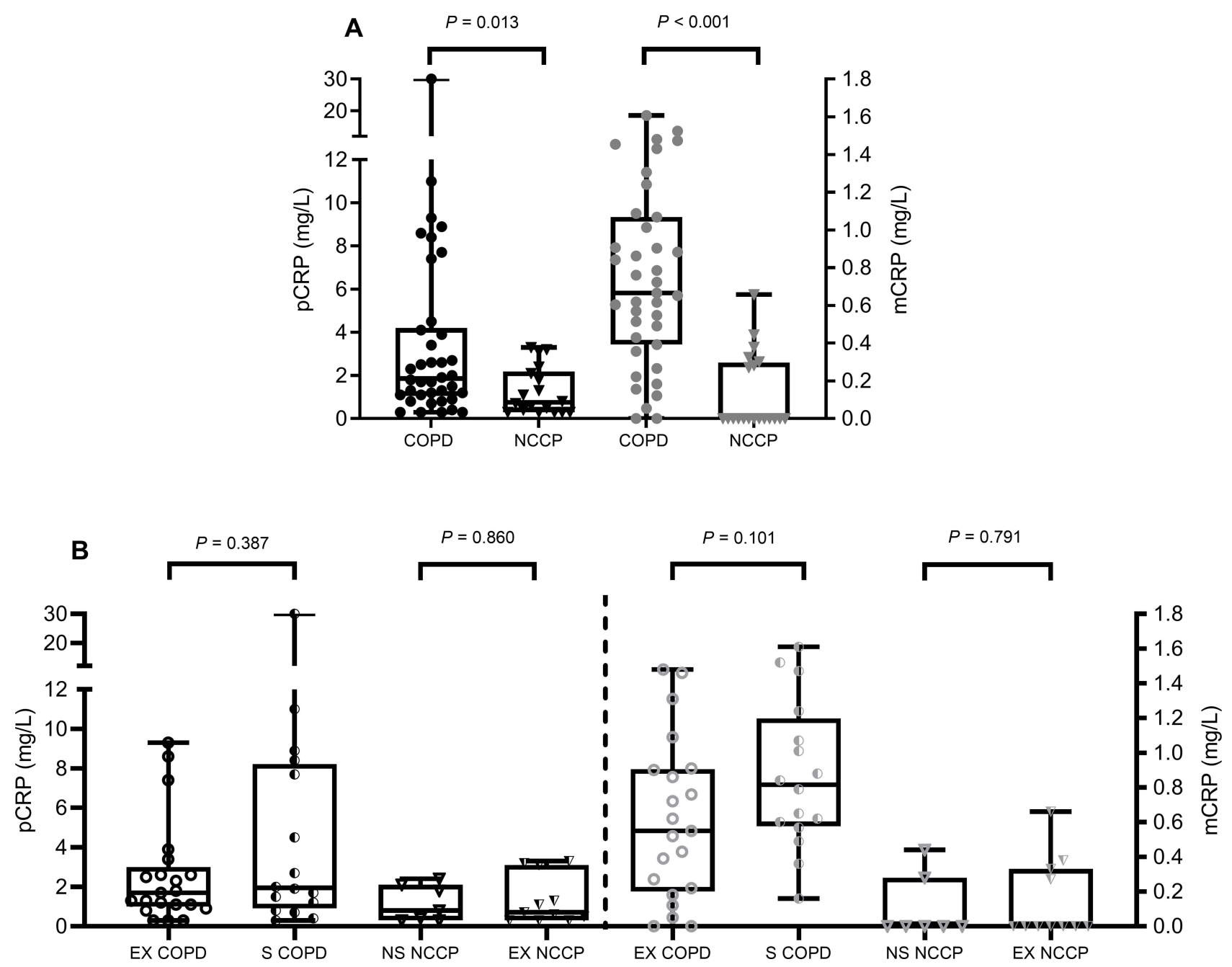

Figure I (A) pCRP (left y-axis; black) and mCRP (right y-axis; grey) levels in 38 patients with COPD (closed circles) and I8 age/gender matched NCCP (closed triangles). (B) PCRP (left y-axis; black) and mCRP (right y-axis; grey) levels in patients with COPD who are ex-smokers (EX COPD; open circles) or current smokers (S COPD; halfopen circles) and NCCPs who are non-smokers (NS NCCP; open triangles) or ex-smokers (EX NCCP; half-open triangles).

Abbreviations: COPD, Chronic Obstructive Pulmonary Disease; NCCP, Non-COPD Control Persons; pCRP, pentameric C-reactive Protein; mCRP, monomeric C-reactive Protein; mg/L, milligram per litre.

mCRP may serve as a potential biomarker to identify early low-grade chronic inflammation in patients with COPD. The current findings need corroboration in larger samples and the investigation of mCRP's associations with other inflammatory markers is warranted. A limitation of the study is the lack of healthy smoking controls. Though the sub-analysis shows no significant effect of smoking on mCRP level, studies have indicated that smoking increases platelet aggregation, which plays an important role in dissociation of pCRP to mCRP. ${ }^{5}$ Therefore, it is important to include healthy smoking controls and non-smoking patients with COPD in a larger future study to analyse the precise diagnostic value of $\mathrm{mCRP}$ in patients with COPD. Research indicated that CRP levels did not differ significantly according to smoking status or biomass exposure, and whether mCRP levels are influenced by different exposures remains to be elucidated. ${ }^{6}$

Several studies have been conducted to assess the efficacy of inhaled therapies which is the mainstay of COPD treatment. pCRP levels were found to be reduced following treatment with inhaled corticosteroids. ${ }^{7}$ However, it remains currently unknown whether and to what extent mCRP transiently increases before, during and/or after a COPD-related exacerbation, and respiratory or other drug therapy can reduce elevated mCRP. To conclude, mCRP is significantly elevated in patients with COPD in comparison to age/sex matched NCCP and 
may be considered as a potential biomarker of chronic low-grade systemic inflammation.

\section{Data Sharing Statement}

Individual participant data that underlie the results reported in this research letter after de-identification (text, figure and table), study protocol and informed consent form can be shared immediately following publication. No end date included. Data can be shared with researchers who provide a methodologically sound proposal to achieve aims in the approved proposal. Proposals should be directed to luc. michiels@uhasselt.be. To gain access, data requestors will need to sign a data access agreement.

\section{Acknowledgments}

The authors acknowledge Ms. Bogaers A. of Hasselt University (Diepenbeek, Belgium) for performing blood sampling and DXA scanning. The UBiLim (University Biobank Limburg; Belgium) is acknowledged as they provided storage and release of all human biological material used in this publication. (Linsen L. et al., Raising to the Challenge: Building a Federated Biobank to Accelerate Translational Research-The University Biobank Limburg. Front Med (Lausanne), 2019. 6: p. 224.)

\section{Funding}

De Brandt J. is funded by the Flemish government. The Research of FWO Aspirant De Brandt J. is sponsored by FWO-grant \#11B4718N. Burtin C. is funded by Limburgs Kankerfonds.

\section{Disclosure}

Ms Revathy Munuswamy and Prof. Dr. Luc Michiels report a patent EP20212664 pending to Luc Michiels, UHasselt. The authors report no other conflicts of interest in this work.

\section{References}

1. Agustí A, Edwards LD, Rennard SI, et al. Persistent systemic inflammation is associated with poor clinical outcomes in COPD: a novel phenotype. PLoS One. 2012;7(5):e37483. doi:10.1371/journal. pone. 0037483

2. Trial J, Potempa LA, Entman ML. The role of C-reactive protein in innate and acquired inflammation: new perspectives. Inflamm Cell Signal. 2016;3(2).

3. Hurst JR, Donaldson GC, Perera WR, et al. Use of plasma biomarkers at exacerbation of chronic obstructive pulmonary disease. Am J Respir Crit Care Med. 2006;174(8):867-874. doi:10.1164/rccm.200604$506 \mathrm{OC}$

4. Leuzzi G, Galeone C, Taverna F, et al. C-reactive protein level predicts mortality in COPD: a systematic review and meta-analysis. Eur Respir Rev. 2017;26(143):160070. doi:10.1183/16000617.0070-2016

5. de la Torre R, Peña E, Vilahur G, et al. Monomerization of C-reactive protein requires glycoprotein IIb-IIIa activation: pentraxins and platelet deposition. $J$ Thromb Haemost. 2013;11(11):2048-2058. doi:10.1111/jth. 12415

6. Aksu F, Capan N, Aksu K, et al. C-reactive protein levels are raised in stable chronic obstructive pulmonary disease patients independent of smoking behavior and biomass exposure. J Thorac Dis. 2013;5 (4):414-421.

7. Perng D-W, Tao C-W, Su K-C, et al. Anti-inflammatory effects of salmeterol/fluticasone, tiotropium/fluticasone or tiotropium in COPD. Eur Respir J. 2009;33(4):778-784. doi:10.1183/09031936.00115308
Journal of Inflammation Research

\section{Publish your work in this journal}

The Journal of Inflammation Research is an international, peerreviewed open-access journal that welcomes laboratory and clinical findings on the molecular basis, cell biology and pharmacology of inflammation including original research, reviews, symposium reports, hypothesis formation and commentaries on: acute/chronic inflammation; mediators of inflammation; cellular processes; molecular mechanisms; pharmacology and novel anti-inflammatory drugs; clinical conditions involving inflammation. The manuscript management system is completely online and includes a very quick and fair peerreview system. Visit http://www.dovepress.com/testimonials.php to read real quotes from published authors. 